RICYDE. Revista Internacional de Ciencias del Deporte doi: $10.5232 /$ ricyde

Rev. int. cienc. deporte

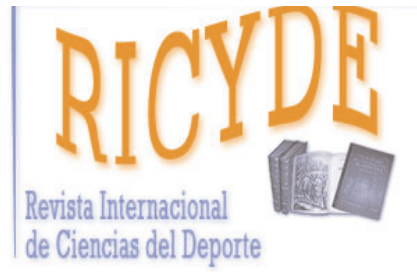

RICYDE. Revista Internacional de Ciencias del Deporte VOLUMEN XII - AÑO XII

Paginas:48-64 ISSN:1885-3137

Número 43 - Enero - 2016

\title{
El apoyo a la autonomía en practicantes de centros deportivos y de fitness para aumentar su motivación \\ The importance of the support to the autonomy in practitioners of sports centers and fitness to increase its motivation and adhesion
}

\author{
Sílvia Puigarnau, Oleguer Camerino, Marta Castañer, Queralt Prat, y M. Teresa Anguera \\ Laboratorio de Observación de la Motricidad. INEFC-Universidad de Lleida. España
}

\section{Resumen}

El objetivo de este estudio exploratorio es analizar las opiniones cualitativas y las percepciones de 56 participantes de un programa de actividad física Basado en la autonomía de seis meses de duración, en un centro de fitness. Pretendemos constatar los beneficios, las barreras, el rol del profesional y la importancia de las relaciones sociales establecidas para mejorar su motivación. Los participantes, 56 adultos sedentarios ( 8 hombres y 48 mujeres) de edades comprendidas entre 18 y 60 años $(M=36,02 ; D T=10,15)$, siguieron las recomendaciones de la OMS de 150 minutos de ejercicio semanal de intensidad moderada y fueron divididos en dos grupos: uno de práctica libre y otro dirigido por un profesional con un estilo de apoyo a la autonomía. Las impresiones sobre los efectos del programa se registraron mediante un diario después de cada sesión y unas entrevistas semiestructuradas realizadas sobre una muestra de los participantes. Los resultados se analizaron siguiendo un diseño de mixed methods de triangulación cuantitativo-cualitativo. El análisis de contendido de todas las transcripciones se realizó con el Software Atlas-ti 6.2 y dio paso a una comparación de proporciones con el Software Statgraphics v.16 y a una interpretación cualitativa de estas unidades temáticas en cada grupo. Los resultados muestran que el apoyo del profesional y la cohesión del grupo han ocasionado una mayor asistencia e interés en las sesiones dirigidas, reduciendo el efecto negativo de las propias barreras. El estilo motivante y autónomo del profesional y la percepción de los beneficios que la actividad física ha aportado a ambos grupos, no han sido suficientes para crear una adherencia a esta actividad física una vez finalizada la intervención; pero sí que constituyen un potente facilitador en la consecución de los objetivos del grupo dirigido que ha mantenido un nivel mayor de compromiso en el programa.

Palabras clave: autonomía; teoría de la autodeterminación (TAD); métodos mixtos.

\section{Abstract}

The aim of this exploratory study was to analyze the qualitative opinions and perceptions of adults who participated in a 6-month exercise motivation program at a fitness center to investigate perceived benefits and barriers, the role of the group leader, and the importance of social relationships to improve motivation. The participants were 56 sedentary adults ( 8 men and 48 women) aged between 18 and 60 years (mean \pm SD age, $36.02 \pm 10.15$ ). Based on the recommendations of the World Health Organization for physical activity in adults, the program consisted of 150 minutes of moderate exercise a week and the participants were divided into two groups: a free group and a guided group aided by autonomy-support strategies. The participants recorded their impressions of the effects of the program in a diary after each session and semi-structured interviews were also held with a eight $(n=8)$ participants. The results were analyzed using a mixed methods design with a triangulation model combining qualitative and quantitative data. The diary entries and interview transcripts were coded using criteria derived from the theoretical framework and analyzed in Atlas-ti 6.2. The resulting quantitative data was analized through a comparison of proportions in Statgraphics, v.16, followed by qualitative interpretation of the thematic units for each group. Our results show that support from the group leader and group cohesion were associated with better exercise adherence and interest in the guided group and reduced the negative effects of barriers to exercising. Neither the motivating, autonomy-supportive style of the group leader nor the perceived benefits of exercising were sufficient to ensure exercise adherence after the program. These factors, however, did have a positive impact on the attainment of goals during the program in the guided group, which showed a greater level of commitment throughout.

Key words: autonomy; self-determination theory (SDT); mixed methods.

Correspondence/correspondencia: Oleguer Camerino

Laboratorio de Observación de la Motricidad. INEFC-Universidad de Lleida. España

Email: ocamerino@inefc.es 
Puigarnau, S.; Camerino, O.; Castañer, M.; Prat, Q.; Anguera, M.T. (2016). El apoyo a la autonomía en practicantes de centros deportivos y de fitness para aumentar su motivación. RICYDE. Revista internacional de ciencias del deporte, 43(12), 48-64. http://dx.doi.org/10.5232/ricyde2016.04303

\section{Introducción}

A nte la evidencia de los beneficios que comporta la práctica de ejercicio físico (Puente y Anshel, 2010; Sallis y Owen, 1999), instituciones públicas y privadas inician múltiples campañas para captar nuevos usuarios. A pesar de tener al alcance estas iniciativas, muchos de los nuevos practicantes son incapaces de mantener su participación voluntaria y autónoma en los programas que ofrecen dichas campañas (Moreno, Águila y Borges, 2011). El sector del fitness se ha convertido en un gran mundo empresarial que no presta suficiente atención al desarrollo de estrategias que potencien hábitos saludables de consolidación autónoma a la actividad física, que asegura una continuidad de la práctica física regular.

Los factores del entorno pueden influir en nuestros comportamientos frente a la actividad física, proporcionándonos diversión, bienestar, vitalidad y generando una autonomía que puede derivar, en algunos casos, hacia la práctica regular de ejercicio físico, o por lo contrario, al abandono prematuro de las actividades deportivas (Bartholomew, Ntoumanis, Ryan, Bosch y Thogersen-Ntoumani, 2011; Standage, Duda y Ntoumanis, 2005). ¿Cómo generar una capacidad de decisión hacia la actividad física? ¿Cómo ayudar a favorecer la iniciativa del participante con un alto grado de capacidad de elección hacia esta actividad física?

La Teoría de la Autodeterminación (TAD) (Deci y Ryan, 1985, 1991, 2000) sostiene que la autonomía es la capacidad de previsión propia hacia una toma de decisiones de iniciativa personal, esencial para desarrollar un proceso de interiorización de la conducta (Williams, Lynch, McGregor, Ryan, Sharp y Deci, 2006; Williams, McGregor, Sharp, Levesque, Kouides, y Ryan, 2006). A su vez, considera que es relevante la competencia en el desempeño y la relación social establecida en el inicio de una actividad física, factores que van perdiendo fuerza a lo largo del proceso de interiorización (Rhaman, Thogersen-Ntoumani, Thatcher y Doust, 2011).

La actuación de los profesionales y técnicos deportivos en los centros de fitness basados en el apoyo a la autonomía con fomento de la toma de decisiones del practicante, puede tener una gran influencia en: el aumento de una motivación autodeterminada (Moustaka, Vlachopoulos, Kabitsis y Theodorakis, 2012), la satisfacción de las necesidades psicológicas básicas, entendida como la satisfacción de la competencia, la autonomía y la relación social (Álvarez, Balaguer, Castillo y Duda, 2009) y la consecuente adherencia a la actividad física a partir de la evolución en los estados de cambio de la conducta de los usuarios transformada en hábitos (Prochaska y Marcus, 1994).

La comunicación de los profesionales de fitness y su estilo de interacción interpersonal sobre los participantes tienen un efecto directo y positivo sobre su grado de satisfacción y puede incentivar una motivación autodeterminada que haga despertar mayor intención de práctica autónoma (Hagger, Chatzisarantis, y Culverhouse, 2003; Jolly y col., 2009; Standage, Duda y Ntoumanis, 2003). Conroy, Coatsworth (2007) y Ommundsen y Kvalo-Eikanger (2007) concluyen que el interés y la diversión de los participantes en actividades físicas aumentan cuando el instructor es capaz de desarrollar un estilo de intervención de soporte y de apoyo a la autonomía.

Nuestro objetivo es realizar un análisis exploratorio, en un centro de fitness y de las opiniones y percepciones que nos han proporcionado los participantes a un programa de incentivación hacia la Actividad Física, con una orientación hacia el ocio y la salud, que sigue las recomendaciones de la OMS de 150 minutos de ejercicio semanales de intensidad moderada. Pretendemos comparar las percepciones de participantes, adultos y sedentarios, distribuidos en dos grupos; uno de práctica libre, sin orientación profesional más allà que la ofertada por el 
Puigarnau, S.; Camerino, O.; Castañer, M.; Prat, Q.; Anguera, M.T. (2016). El apoyo a la autonomía en practicantes de centros deportivos y de fitness para aumentar su motivación. RICYDE. Revista internacional de ciencias del deporte, 43(12), 48-64. http://dx.doi.org/10.5232/ricyde2016.04303

centro de fitness; y otro dirigido por profesionales con un estilo y recursos estratégicos con apoyo a la autonomía. Queremos resaltar la eficacia de las estrategias pedagógicas que aplican los profesionales sobre la motivación y la satisfacción de las necesidades básicas según la Teoría de la Autodeterminación (TAD). Estas evidencias pueden permitir a educadores, promotores $\mathrm{y}$ directores deportivos fomentar actitudes $\mathrm{y}$ estrategias para una mayor satisfacción de los usuarios en sus servicios deportivos.

\section{Método}

La presente investigación se enmarca en el enfoque mixed methods en el ámbito de las Ciencias de la Actividad Física y el deporte (Angura, Camerino, Castañer y Sánchez-Algarra, 2014). Planteamos una variante del diseño de triangulación (Creswell y Plano Clark, 2007, p 67), caracterizado por un procedimiento bietápico cuantitativo-cualitativo que constituye un estudio de casos de tipo intrínseco e instrumental (Stake, 1994, 1998; Gerring, 2007) de carácter revelador (Yin, 2014).

\section{Participantes}

La muestra del estudio estuvo formada por un total de 56 participantes adultos ( 8 hombres y 48 mujeres); con edades comprendidas entre 18 y 60 años $(M=36,02 ; D T=10,15)$ y seleccionados a partir de un muestreo aleatorio de personas voluntarias e integrantes de asociaciones culturales de una ciudad de la provincia de Lleida. El criterio de inclusión consistía en que los participantes eran sedentarios y se encontraban en un estadio de cambio contemplativo, de preparación o de acción, según la Teoría Transteórica (Prochaska y Diclemente, 1983), que implica la intención de iniciarse en la práctica deportiva o que su práctica es inferior a las mínimas recomendadas de 150 minutos de ejercicio semanal de intensidad moderada (Prochaska y Marcus, 1994). Después de una revisión médica, se utilizó como criterio de exclusión el padecer alguna enfermedad o estar en tratamiento farmacológico incompatible.

Los participantes firmaron un consentimiento informado y de confidencialidad (Anexo 1), en el que se especificaban las 3 sesiones semanales del programa y las condiciones del estudio. Dividimos los participantes en dos grupos. El primer grupo, dirigido siempre por el mismo profesional con estrategias de apoyo a la autonomía, estaba formado por 35 adultos de edades entre 18 y 56 años $(M=38,83 ; D T=9,48)$ quienes tenían que asistir a dos sesiones semanales dirigidas en el centro deportivo y una sesión libre. El segundo grupo, de práctica libre y con las mismas condiciones que cualquier usuario del centro de fitness, estaba compuesto por 21 adultos de edades entre 21 y 60 años $(M=31,86 ; D T=9,88)$ quienes debían cumplir las 3 sesiones semanales de 150 minutos de ejercicio de intensidad moderada, pero sin el seguimiento exclusivo de un profesional.

\section{Instrumentos}

Previamente a la administración del programa, se seleccionó a los participantes aplicando el cuestionario de Estado de Cambio que consiste en la catalogación de seis diferentes estados de cambio comportamental (Prochaska y DiClemente, 1983; Prochaska, DiClemente y Norcross, 1992). En el desarrollo del programa se ha utilizado una combinación de instrumentos - diario y entrevista- cualitativos, diseñados con dimensiones coincidentes con el objetivo de conseguir la complementariedad de la información y administrados a lo largo del programa:

- Diario pautado (Surbeck, Han y Moyer, 1991; Zabalza, 2008). Se elaboró con dimensiones derivadas del marco teórico (Deci y Ryan, 2000) (Tabla 1) para captar las motivaciones, 
Puigarnau, S.; Camerino, O.; Castañer, M.; Prat, Q.; Anguera, M.T. (2016). El apoyo a la autonomía en practicantes de centros deportivos y de fitness para aumentar su motivación. RICYDE. Revista internacional de ciencias del deporte, 43(12), 48-64. http://dx.doi.org/10.5232/ricyde2016.04303

percepciones y dificultades en el desarrollo de cada sesión, siendo equiparables a las dimensiones de la entrevista.

Tabla 1. Dimensiones del diario pautado.

- Motivos de asistencia a la sesión

- Sensaciones antes, durante y después de la sesión

- Nivel de consecución de las actividades elegidas o indicadas por el profesional

- Aspectos destacados del profesor y de los compañeros

- Dificultades encontradas y superación de las mismas

- Entrevista semiestructurada. Se construyó un guión de entrevista orientado por el objetivo del estudio y compuesto por seis dimensiones desarrolladas en diferentes preguntas sobre: la trayectoria deportiva, la superación de barreras, la satisfacción de necesidades, la percepción de beneficios aportados antes-durante-después de las sesiones y sus intenciones. El guión de la entrevista (Tabla 2) fue sometido a una validación por un panel de expertos de gestores y animadores deportivos en el cual se comprobó la univocidad, pertinencia e importancia de las preguntas para ser autentificado en una prueba piloto y poderse administrar a la muestra seleccionada (Wilson, Kaplan, CrawFort y Robert, 2007).

Tabla 2. Guión de dimensiones de la entrevista.

\footnotetext{
Historia de práctica deportiva

- Antecedentes de práctica deportiva familiar o de amistades e itinerario personal

- Motivos, predisposición y expectativa inicial de la participación en el programa

Barreras y dificultades en el seguimiento del programa

- Aprovechamiento de los recursos del programa o de otros personales

- Inconvenientes, impedimentos y dudas en el seguimiento del programa

- Estrategias de superación de las dificultades del programa

- Razones de abandono o no cumplimiento del programa

Percepciones previas a las sesiones

- Sensaciones y expectativas personales previas a la sesión

- Influencias iniciales de las condiciones del espacio y/o el material

- Influencia del técnico deportivo y de los compañeros en la elección del tipo de sesión

Percepciones durante las sesiones

- Impresiones y estrategias motivacionales de superación en el transcurso de las sesiones

- Condiciones de facilitación en el desarrollo del espacio y/o el material

- Elementos de facilitación en el desarrollo de técnicos deportivos y compañeros

Percepciones después de las sesiones

- Emociones y sensaciones de satisfacción o insatisfacción al finalizar la sesión

- Evocación del resultado y satisfacciones, sensaciones de mejora posteriores

- Grado de cumplimiento de las expectativas iniciales sobre el programa

Intenciones

- Grado de cumplimiento de las expectativas y demandas iniciales sobre el programa

- Intenciones, expectativas y objetivos futuros de continuidad de práctica deportiva

- Posibles cambios y ajustes de hábitos de vida derivados del programa
}

\section{Procedimiento}

El programa de incentivación hacia la actividad física se desarrolló de febrero a julio de 2014 en una instalación deportiva de una ciudad de la provincia de Lleida. Se facilitó el acceso a la totalidad de los participantes del estudio que se comprometieron a seguirlo, con diferentes características según el grupo, durante seis meses, con una asistencia del $80 \%$ a las tres sesiones semanales de 50 minutos de duración. Todos los participantes, tanto del grupo libre como el dirigido, podían utilizar todas las instalaciones y recursos materiales disponibles 
Puigarnau, S.; Camerino, O.; Castañer, M.; Prat, Q.; Anguera, M.T. (2016). El apoyo a la autonomía en practicantes de centros deportivos y de fitness para aumentar su motivación. RICYDE. Revista internacional de ciencias del deporte, 43(12), 48-64. http://dx.doi.org/10.5232/ricyde2016.04303

según sus horarios. Las sesiones dirigidas de los grupos fijos constaban de una preacción, un desarrollo y unos estiramientos finales para experimentar las diferentes disciplinas de fitness así cómo las técnicas de pilates, ciclismo indoor, entrenamiento funcional, yoga, tonificación, body pump, body combat, etc. Además experimentaron con deportes como Pádel y otros colectivos como el Hockey, el Básquet, el Fútbol o Juegos de colaboración, colaboraciónoposición y de oposición.

Las sesiones a las que podían asistir el grupo de funcionamiento libre eran las propias ofertadas por un centro deportivo, abiertas a la totalidad de los usuarios de las instalaciones colaboradoras.

Las premisas de las sesiones fueron elaboradas siguiendo los principios y orientaciones del estilo de apoyo a la autonomía:

- Sesiones de intensidad moderada y organizadas según objetivos.

- Incentivar formas de motivación intrínseca autodeterminada y de satisfacción de las necesidades básicas como la autonomía, la competencia y la relación social, (Teoría de la autodeterminación, TAD).

- Clases variadas, divertidas y poco monótonas con contenidos en busca de la competencia, la autonomía y la seguridad.

- Sesiones adaptadas al nivel de los participantes basada en preferencias.

- Incentivación de la práctica deportiva fuera de las sesiones organizadas.

- Promoción de la interacción y cohesión de grupo.

Los diarios de impresiones fueron cumplimentados por cada participante inmediatamente después de cada una de las 75 sesiones en el mismo centro deportivo. Para garantizar la representatividad de diferentes opiniones en las entrevistas se seleccionó a ocho participantes representativos de entre los 21 y 35, que habían superado los cuatro meses de asistencia regular en el programa, cuatro del grupo dirigido y cuatro del grupo libre. Las entrevistas se grabaron con el debido consentimiento firmado para posteriormente ser trasncritas literalmente.

Las transcripciones de los diarios y de las entrevistas fueron sometidas a un análisis de contenido con el programa Atlas-ti 6.2 (Scientific Software Development GmbH, Berlin) y segmentando la información en unidades temáticas, mediante la opción de codificación libre, según el marco teórico de este estudio (Puente y Anshel, 2010).

Se llevó a cabo un tratamiento estadístico de comparación de proporciones de los datos, mediante el programa Statgraphics Centurion, v. 16 (StatPoint Technologies, Inc.), que permite realizar una prueba binomial a cada par de ratios (del grupo dirigido y del grupo libre) ponderadas en función del tamaño de las submuestras.

En una segunda etapa, los resultados del análisis de contenido fueron analizados cualitativamente para comparar los grupos según las unidades textuales, con el fin de clasificar e interpretar la información de los objetivos del estudio. 
Puigarnau, S.; Camerino, O.; Castañer, M.; Prat, Q.; Anguera, M.T. (2016). El apoyo a la autonomía en practicantes de centros deportivos y de fitness para aumentar su motivación. RICYDE. Revista internacional de ciencias del deporte, 43(12), 48-64. http://dx.doi.org/10.5232/ricyde2016.04303

\section{Resultados}

\section{Primera etapa: Tratamiento cuantitativo}

A partir de las agrupaciones realizadas y del recuento de las respectivas frecuencias para cada unidad temática y subtema, se ha efectuado una comparación entre las respuestas obtenidas por los participantes de ambos grupos. El análisis de comparación de proporciones de las frecuencias de las opiniones de los dos grupos de los diarios y entrevistas, nos señala que los resultados del grupo dirigido son superiores a los del grupo libre (Tabla 3).

Tabla 3. Comparación de proporciones por subtemas entre Grupo Dirigido y Grupo Libre.

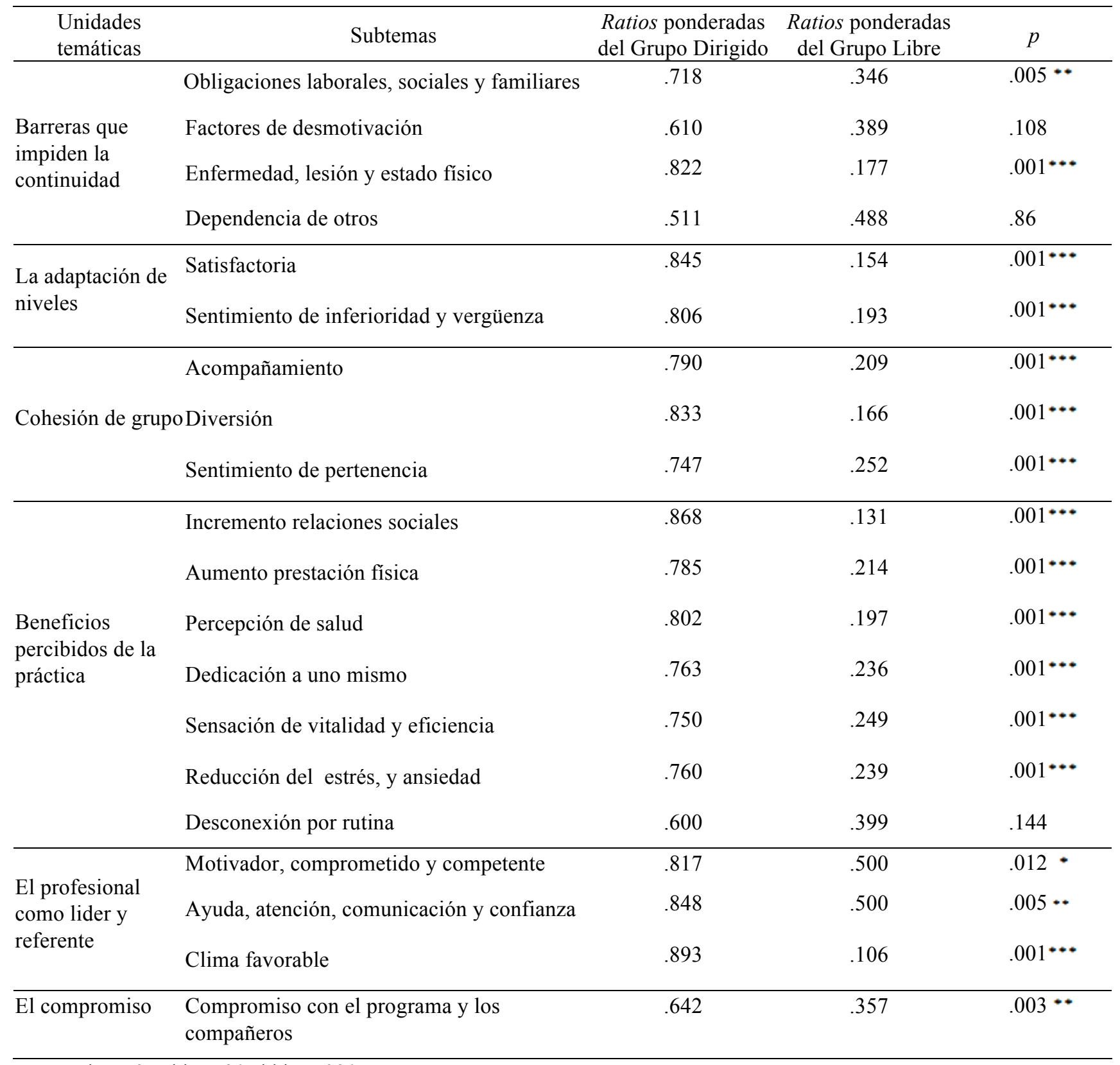

Nota: $* p<.05 ; * * p<.01 ; * * * p<.001$.

Estos valores los expresamos en un mapa conceptual (Figura 1) en el que insertamos los valores $p$ de la prueba binomial de la comparación de proporciones y en el que también expresamos mediante conectores la relación de las unidades temáticas y subtemas encontrados. El mapa muestra que las valoraciones más sobresalientes y significativas del 
Puigarnau, S.; Camerino, O.; Castañer, M.; Prat, Q.; Anguera, M.T. (2016). El apoyo a la autonomía en practicantes de centros deportivos y de fitness para aumentar su motivación. RICYDE. Revista internacional de ciencias del deporte, 43(12), 48-64. http://dx.doi.org/10.5232/ricyde2016.04303

grupo dirigido en comparación al grupo libre son: las barreras de enfermedades y lesiones y los sentimientos de inferioridad y vergüenza. También podemos ver que los beneficios más percibidos por el grupo dirigido son: la prevención de salud, la dedicación de uno mismo, la sensación de vitalidad y la reducción del estrés. Y finalmente, al profesional como motivador comprometido capaz de ofrecer ayuda y confianza.

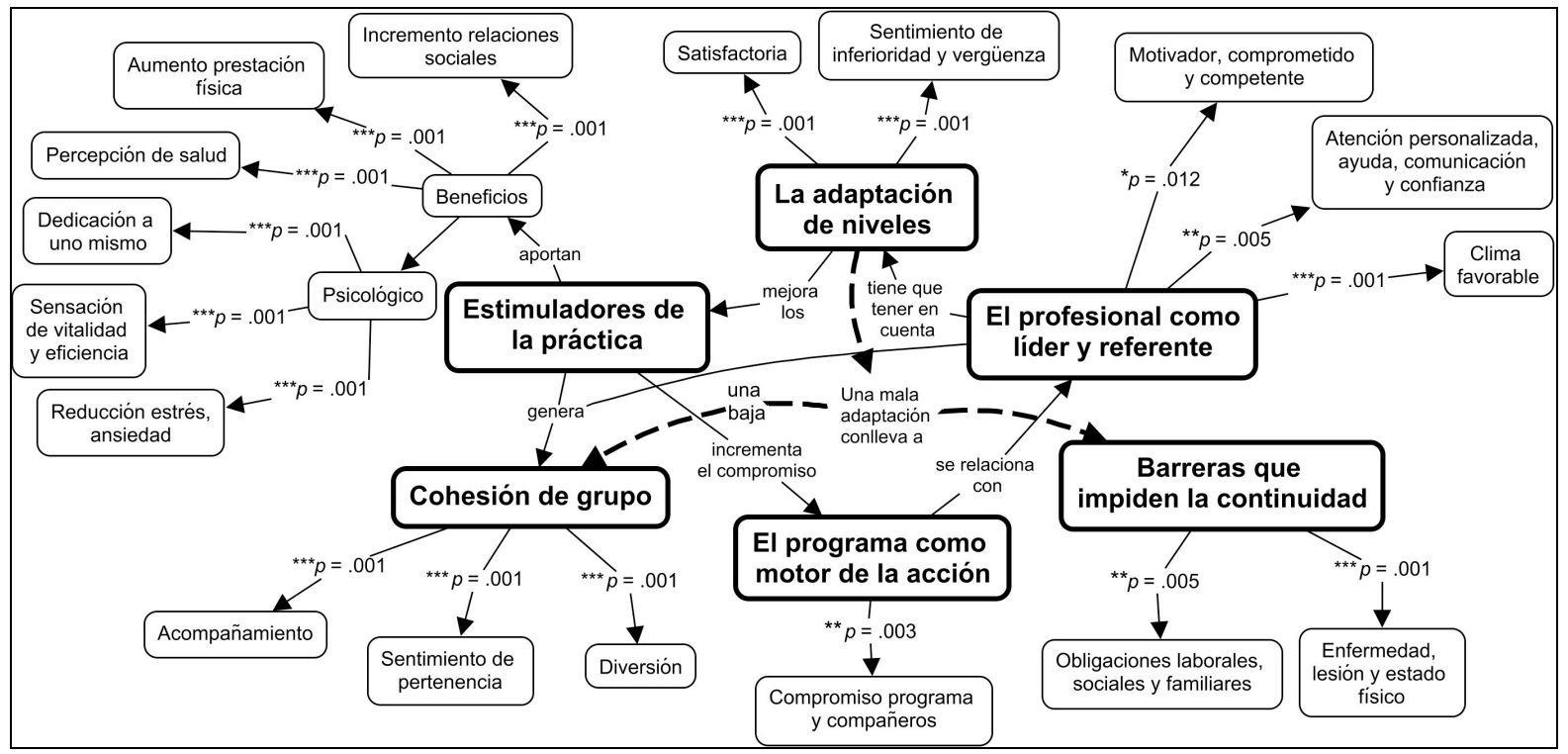

Nota: $* p<.05 ; * * p<.01 ; * * * p<.001$.

Figura 1. Mapa conceptual de la relación entre los diferentes unidaes temáicas y subtemas encontrados.

\section{Segunda etapa: Tratamiento cualitativo}

En la segunda etapa de análisis cualitativo comparamos las opiniones de los dos grupos sobre: las dificultades, satisfacciones, ayudas y motivaciones y barreras que viven los participantes a lo largo del programa, con aportaciones textuales representativas y la referencia del grupo del cual proceden.

\section{A. Barreras que impiden la continuidad}

Aunque el grupo de funcionamiento libre cumplió menos con las sesiones semanales propuestas, son los participantes del grupo dirigido quiénes expresan mayores barreras de impedimento en el seguimiento del programa. Los dos grupos exiben el cansancio, las obligaciones de trabajo y familia, el no disponer de instructor o compañía y la pereza como las principales barreras. La desmotivación y el cansancio son dificultades que aparecen frecuentemente, y que los participantes del grupo dirigido vencen al ser conscientes de que alguien les está esperando.

"No sé si es el tiempo pero últimamente no tengo nada de ganas de asistir, no tengo ganas de hacer nada. Y los viernes... aún menos. (Grupo dirigido_3CM)

Los estados psicológicos negativos previos y durante la práctica suponen un inconveniente. La percepción de no competencia e inseguridad y el sentimiento de ridículo se superan mejor con la ayuda del profesional y del grupo.

"No sé porque, a veces, tengo miedo de lo que haremos, por si podré seguir, por si aguantaré... soy la mayor de todas y no quiero hacer el ridículo". (Grupo dirigido_4DG) 
Puigarnau, S.; Camerino, O.; Castañer, M.; Prat, Q.; Anguera, M.T. (2016). El apoyo a la autonomía en practicantes de centros deportivos y de fitness para aumentar su motivación. RICYDE. Revista internacional de ciencias del deporte, 43(12), 48-64. http://dx.doi.org/10.5232/ricyde2016.04303

En el grupo dirigido aparecen mecanismos de culpabilidad si no pueden mantener la continuidad. El profesional crea un cierto compromiso y constituye un acicate positivo hacia la consecución de las expectativas iniciales marcadas.

“Tenía mucha pereza y sensación de que perdería el tiempo porque mi marido tiene mucho trabajo. Pero todas van y no puedo fallar ahora. Además me pasan a buscar, pero, por ganas, no iría. (Grupo dirigido_2CG)

Los participantes del grupo dirigido, expresan la dependencia que tienen con el profesional que les motiva a seguir con las recomendaciones dadas. Los participantes de los dos grupos, también manifiestan que una vez finalizado el programa no serán capaces de seguir si nadie les exige.

"Sola... hacer las recomendaciones mínimas cuando se acabe, imposible, necesito a alguien que me ayude como mínimo para empezar. Lo que es más difícil para mí es decidirme y si tengo que trabajar duro... necesito a alguien que me exija". (Grupo dirigido_2MB)

"Es mucho mejor que alguna persona te dirija la sesión, el rendimiento es mucho más alto. La motivación que te saca el instructor es mucho más alta". (Grupo libre_9JMP)

\section{B. La adaptación de niveles}

Participar en un grupo con características heterogéneas y de diferentes niveles, puede facilitar la adaptación y el trabajo colectivo, pero a la vez puede conllevar frustración, comparación, vergüenza o sentimientos de inferioridad. En los grupos dirigidos se ha destacado positivamente la capacidad del profesional para conseguir que el trabajo sea adaptado a las características de cada uno de ellos. En esta línea de actuación el grupo libre que asiste a las clases dirigidas, también valora positivamente que puedan participar personas con diferentes niveles.

"Me gusta que a mí y a otras compañeras que podemos realizar más esfuerzo, nos pida ejercicios de más dificultad. Así todas podemos trabajar a nuestro nivel. De esta manera puedo hacer un esfuerzo más alto que si seguimos todos el mismo patrón“. (Grupo dirigido_6IG)

"No he podido realizar algún ejercicio pero lo ha entendido perfectamente. Me ha adaptado a los ejercicios y me ha ido corrigiendo constantemente porque éramos pocos en clase“. (Grupo libre_6GB)

A su vez, la adaptación de nivel es uno de los aspectos que más negativamente se ha valorado cuando la percepción del participante ha sido de inferioridad respecto al grupo.

"No me ha gustado correr porque tenía miedo a ser la única que no pudiese seguir el ritmo. No me gusta nada quedarme atrás y menos ser menos". (Grupo libre_8MARB)

No sólo los participantes de menor nivel son los que pueden asimilar malas sensaciones, también los participantes de mayor competencia, pueden sentir que no trabajan suficiente o que podían haber aprovechado más el tiempo. Por esta razón, se debe procurar que todos trabajen a su nivel para no aburrir o desmotivar.

"Me gustaría que nos exigieran más y así las sesiones serían más duras. Ahora vamos al ritmo de las que se quejan". (Grupo dirigido_13OF)

Esta situación se puede dar fuera de las clases dirigidas en las que no intervienen directamente los profesionales pero pueden influir aportando recomendaciones a aquellas personas que lo soliciten. 
Puigarnau, S.; Camerino, O.; Castañer, M.; Prat, Q.; Anguera, M.T. (2016). El apoyo a la autonomía en practicantes de centros deportivos y de fitness para aumentar su motivación. RICYDE. Revista internacional de ciencias del deporte, 43(12), 48-64. http://dx.doi.org/10.5232/ricyde2016.04303

\section{La cohesión de grupo}

Los participantes que se han rodeado de otras personas, han obtenido mejores resultados de asistencia. A pesar de ello, los mejores resultados aparecen cuando se consigue cohesión de grupo y sentimiento de pertenencia a este. de pertenencia. Esto es promocionado y propiciado por el profesional.

"Con el grupo me lo paso genial, yo me levanto esperando ir, la verdad es que no me importa levantarme a las seis de la mañana porque sé que voy a pasar un buen rato". (Grupo dirigido_8MC)

Los participantes del grupo libre no expresan los beneficios de la cohesión de forma tan clara, manifestando que practicar con otra gente es mucho más divertido, y que en ocasiones, es imprescindible para iniciar la práctica. Por esta razón el grupo de actividad libre que asiste esporádicamente a las clases dirigidas pueden llegar a obtener sensaciones parecidas al grupo dirigido.

"Te sientes más apoyado, compartes las sensaciones con otra gente y es más divertido. Además acabas conociendo nueva gente". (Grupo libre_18NC)

"Realizar ejercicio con alguien siempre es más divertido. Hablas o escuchas las bromas de otros. Al final haces un poco de vida social”. (Grupo libre_2AM)

Llega a tener tal importancia rodearse de otras personas para la práctica deportiva, que la intensidad de esta actividad pasa a estar en segundo plano, siendo la diversión y el compartir la experiencia una prioridad. Los participantes buscan complicidad entre sus compañeros y sentirse apreciados. Cuando conseguimos esta complicidad, interacción y a la vez diversión, aparecen los sentimientos de identificación en un grupo, sean los compañeros del propio grupo dirigido o los amigos o compañeros del grupo libre. Esta identificación en el colectivo conllevará muchos beneficios sociales pero, a la vez, personales, así como la mejora de la autoestima y del autoconcepto.

\section{Beneficios percibidos de la práctica}

Los beneficios percibidos tales como: desconexión de rutinas, incremento de relaciones sociales, aumento de prestación física, dedicación a uno mismo, mejora del ánimo y la salud, son los principales estímulos que empujan a los participantes de los dos grupos a seguir con el programa.

"También me ayuda que aquí consigo no pensar, cosa que no puedo hacer casi nunca. Con el deporte sí que puedo, vaya que es como hacer un reset y va muy bien. Me encanta empezar el día así, ya veo que lo tendré que poner como hábito y no sacarlo". (Grupo libre_1AC)

"Voy porque me lo paso muy bien y después me siento mucho mejor conmigo misma. Durante una hora me dedico a mí misma". (Grupo dirigido_15SC)

"Estoy contenta de haber venido, satisfecha porque noto que mejoro, estoy más fuerte, en forma y por lo tanto más animada cuando acabo". (Grupo libre_19NS)

Es interesante remarcar los beneficios psicológicos que ofrecen satisfacciones inmediatas y que condicionan, la continuidad de la participación al programa. Estar orgulloso de uno mismo, obtener sensación de vitalidad, mejorar la autoestima, reducir el estrés y la ansiedad, son mecanismos intrapsíquicos favorecedores de la actividad física.

"Lo necesitaba. ¡Me he dado cuenta de que saco mucho mi estrés y salgo flotando! Me encanta esta sensación. Salgo con ganas de comerme el mundo. (Grupo dirigido_8MAGB) 
Puigarnau, S.; Camerino, O.; Castañer, M.; Prat, Q.; Anguera, M.T. (2016). El apoyo a la autonomía en practicantes de centros deportivos y de fitness para aumentar su motivación. RICYDE. Revista internacional de ciencias del deporte, 43(12), 48-64. http://dx.doi.org/10.5232/ricyde2016.04303

\section{E. El profesional como lider y referente}

Las actitudes del profesional que más son valoradas por el grupo dirigido son la seguridad, la paciencia y la actitud de motivación. Además, destacan la ayuda y la atención personalizada que reciben, el interés por su estado diario, la buena organización y la voluntad hacia un clima favorable en las sesiones.

"Nos da una gran confianza. Tiene mucho interés en que estemos a gusto, nos pregunta siempre cómo estamos. Intenta hacer cosas variadas para que todas nos sintamos cómodas. (Grupo dirigido_5DP)

"Yo necesito que haya buen ambiente y buen rollo en una sesión, esto es lo más importante. Un profesor que lo consiga, y haga la sesión amena, lleva a que quieras volver y que te lo pases genial a pesar de cansarte mucho! Por esto voy a estas clases". (Grupo libre_20OF)

Esto supone una especial sensibilidad y una capacidad de liderazgo, energía y disponibilidad por parte del profesional, así como una permanente promoción hacia la práctica de la actividad física en la vida cotidiana.

"No muestra nunca desidia ni cansancio. Siempre nos anima a realizar todo lo que podamos en las sesiones. Nos anima y hace las clases más agradables". (Grupo dirigido_6IG)

"Pone mucho entusiasmo en las actividades y tiene mucha capacidad de innovación, clase tras clase, para motivarnos sin que nos aburramos". (Grupo dirigido_11GC)

Una óptima instrucción debe dar la información clara y a la vez introducir correcciones y aportaciones que provoquen una mejora en los participantes. Al igual que los anteriores, son numerosos los comentarios con relación a cómo se dan los mensajes. Valoran de forma muy positiva las correcciones y una información clara que aporte seguridad en la ejecución de tareas.

\section{F. El compromiso con el programa}

La principal motivación obtenida es la del compromiso adquirido con los requerimientos mínimos del programa y sus expectativas de cambio; el grupo dirigido lo relaciona con el profesional que desarrolla las sesiones y con sus compañeros:

"Muchísimas veces me cuesta venir. Siempre por pereza, porque no tengo ganas. Ya te digo que si no fuese porque cogí el compromiso y porque sé que vosotros me esperáis... ya no vendría. Siempre encuentro alguna excusa para no venir". (Grupo Dirigido_8MARB)

Los practicantes del grupo libre tienen más dificultades para cumplir el compromiso y utilizan estrategias como crearse hábitos de participación en horarios determinados, asistencia a clases dirigidas del gimnasio en forma de rutina semanal o quedar con compañeros para practicar deporte.

"Yo me hubiese estimado más estar en un grupo para obligarme más y tener alguien con quien practicar siempre, pero los horarios que había no me eran muy favorables". (Grupo libre_17MOB)

\section{Discusión}

Los resultados hallados sobre la importancia del grupo y de la actitud del profesional como claves desencadenantes de la práctica deportiva, concuerdan con algunos estudios realizados sobre las estrategias efectivas de los programas de incentivación (Leyton y col., 2013; Silva y col., 2008). La necesidad de una intervención, con orientación hacia la autonomía para mejorar la motivación hacia la práctica de ejercicio físico, aparece en otros estudios en los que se ha observado este fenómeno (Kinnafick, Thogersen-Ntoumani, Duda y Taylor, 2014). 
Puigarnau, S.; Camerino, O.; Castañer, M.; Prat, Q.; Anguera, M.T. (2016). El apoyo a la autonomía en practicantes de centros deportivos y de fitness para aumentar su motivación. RICYDE. Revista internacional de ciencias del deporte, 43(12), 48-64. http://dx.doi.org/10.5232/ricyde2016.04303

Edmunds, Ntoumanis y Duda (2008) analizaron como la actuación de los profesionales aumenta el interés hacia la actividad física; en su estudio demuestran que una intervención en base a la TAD obteniene mejores resultados en las regulaciones autodeterminadas, percepción de autonomía, satisfacción de las necesidades psicológicas básicas y la relación con el resto del grupo. Puente y Anshell (2010), en una investigación con estudiantes universitarios, valoran como podían variar la autonomía y la competencia percibida según el estilo de interacción de los profesores; cuando el estilo era próximo al apoyo de la autonomía, aparecían sentimientos de diversión y aumento de la frecuencia en la práctica de ejercicio físico. Estos estudios corroboran algunos de los resultados obtenidos en el grupo dirigido: el programa, el monitor y la dinámica de grupo, condujeron a los participantes a una alta participación y la superación de las propias barreras (desmotivación, cansancio, aspectos de inseguridad, obligaciones u otras prioridades, citadas por los participantes en sus diarios y entrevistas).

En nuestro estudio obtenemos resultados muy positivos con relación a los factores sociales y sus influencias. Según Vallerand (2001) y Pope y Wilson (2012), los factores sociales que promueven sentimientos de competencia, pueden desarrollar la motivación intrínseca. Consideramos que factores externos como feedback positivos del profesional, atención personalizada, dar buena información o transmitir un buen clima, han promovido sentimientos de competencia según las opiniones y percepciones de los participantes del grupo dirigido, siendo estos aspectos los que han remarcado como motivantes para seguir asistiendo a las clases programadas.

Ng, Ntoumanis, Thogersen-Notumani, Stott y Hindle (2013) concluyeron en su estudio que la calidad de las interacciones entre individuos que se encuentran en un mismo programa, son esenciales para la satisfacción de las necesidades básicas y la adquisición de hábitos saludables. También lo afirman King (2001) y Leyton, et al. (2013), remarcando la importancia del componente social sobre todo en mujeres. De la misma manera, en nuestros resultados podemos observar que el grupo dirigido consiguió una mayor cohesión de grupo, asegurando sentimientos de pertinencia y de diversión que conllevarían una mayor asistencia a las sesiones.

Con estos resultados nos podemos remitir a las afirmaciones de la misma TAD en que Ryan y Deci (2000) remarcan la influencia positiva de la relaciones con otras personas para la obtención de sentimientos de competencia y autonomía. También Vallerand (2001), en su modelo jerárquico, afirma la necesidad que los demás influyan en nuestras motivaciones, corroborando que un estilo de soporte a la autonomía, el cual busca la interacción y la complicidad entre los participantes, debe conllevar influencias positivas hacia la adherencia.

Podemos afirmar que, la actuación del profesional basada en la promoción de las estrategias de la TAD (Moreno y Martínez, 2006) y el grupo actúan como incentivos para la adherencia a la práctica deportiva. Debemos sumar a estos factores los beneficios percibidos de esta práctica, ya que tienen la función de estimuladores al ser la consecuencia de la experiencia vivida por los participantes. Adie, Duda y Ntoumanis (2012) y Vallerand (2007a 2007b) expresan que un estilo de soporte a la autonomía se vincula a: las sensaciones de bienestar, al vínculo social de pertenencia al grupo, la vitalidad, la autoestima, el disfrute, el interés, la concentración, el esfuerzo y la persistencia. En este estudio, obtenemos datos significativos mucho más elevados en el grupo dirigido que en el grupo libre. Los beneficios que perciben son con relación a la mejora de la condición física, la mejora de las relaciones sociales, la percepción de salud y una amplia gama de condiciones de mejora psicológica así como la dedicación a un mismo, la sensación de vitalidad y eficiencia o la reducción de ansiedad y estrés. 
Puigarnau, S.; Camerino, O.; Castañer, M.; Prat, Q.; Anguera, M.T. (2016). El apoyo a la autonomía en practicantes de centros deportivos y de fitness para aumentar su motivación. RICYDE. Revista internacional de ciencias del deporte, 43(12), 48-64. http://dx.doi.org/10.5232/ricyde2016.04303

Debemos también tener en cuenta que en toda intervención aparecerán factores que pueden llegar a entorpecer la adquisición de hábitos de práctica física regular, y pueden ir con relación a la intervención del profesional, de situaciones creadas en el grupo o de las barreras que los participantes no son capaces de solventar. Se ha observado en los comentarios, valoraciones positivas en relación con la capacidad del profesional para adaptar las sesiones a todas las personas y niveles. Pero también hemos obtenido resultados negativos cuando la percepción que tienen los participantes es que el nivel de clase no es adecuado a sus posibilidades, sea por exceso de nivel o por defecto de exigencia.

En relación con las barreras, no podemos dejar de lado la alta incidencia del resto de obligaciones familiares y de trabajo que los participantes tienen. En este tipo de barrera solo podemos actuar ayudándolos a tener una mejor organización de sus horarios para ser más efectivos y poder llegar a una práctica mínima. A pesar de eso, sí que podemos intervenir en aquellas barreras con relación al cansancio, o a estados psicológicos negativos que surgen de las experiencias vividas en las sesiones. Además, debemos incentivar el estilo de instrucción basado en la TAD para evitar situaciones de desmotivación que conllevan un bajo índice de participación por parte de los usuarios. Para evitar que aparezca la desmotivación, Standage, Gillison y Treasure (2007) citan que el verdadero reto del profesional es activar la motivación para llevar a cabo todas las tareas de la mejor manera posible, así como escoger tareas motivadoras y dar protagonismo a los participantes (Chatsizarantis et al., 2003; Condon y Collier, 2002).

\section{Conclusiones}

Este estudio nos proporciona una buena evidencia de la necesidad de una intervención de apoyo hacia la autonomía por parte de los profesionales. El análisis llevado a cabo nos ha permitido constatar que aquellos participantes del grupo dirigido han seguido más las sesiones programadas que aquellos participantes del grupo libre ya que algunos les ha faltado un estímulo o un mayor compromiso. Tal como hemos visto con las percepciones y opiniones de los participantes del grupo dirigido, el profesional es fundamental si posee una serie de condiciones, actitudes y premisas en el desarrollo de sus sesiones. Un estilo de apoyo a la autonomía predispone a la obtención de un mayor protagonismo y participación que nos conducirá a un aumento de la motivación a la práctica física.

Para ello debemos conseguir que los profesionales o técnicos deportivos actúen, con aquellas personas que se inician en la práctica deportiva, con estas recomendaciones:

- Crear compromiso, hacer sentir al participante que es parte importante para el buen funcionamiento de la clase.

- Potenciar un grupo que interactúe para crear vínculos afectivos entre los participantes y así incentivar el compromiso colectivo.

- Actuar como líder, referente, que transmita seguridad, vitalidad, confianza e interés por cada uno de los participantes.

- Fijar objetivos de forma individualizada y objetiva y motivar a cada una de las personas a cumplir sus metas. De esta manera se conseguirá incentivar el sentimiento de competencia.

- Adaptar los ejercicios a los diferentes niveles y grados de exigencia, evitando situaciones en las que se acentúen las diferencias.

- Crear ambientes agradables donde haya diversión, teniendo claro el objetivo de acondicionamiento físico que los participantes buscan. 
Puigarnau, S.; Camerino, O.; Castañer, M.; Prat, Q.; Anguera, M.T. (2016). El apoyo a la autonomía en practicantes de centros deportivos y de fitness para aumentar su motivación. RICYDE. Revista internacional de ciencias del deporte, 43(12), 48-64. http://dx.doi.org/10.5232/ricyde2016.04303

- Organizar las sesiones de manera creativa y clara, fijar metas y ser capaz de transmitirlas de la mejor manera posible. A la vez, se deben proponer actividades de acuerdo con los gustos de los participantes y que incentiven la interacción positiva entre los mismos participantes y entre el profesional y los participantes.

- Dar información clara, dar feedback adecuado a cada persona y aportarle conocimiento para potenciar la autonomía.

Con esta actuación de los profesionales de apoyo a la autonomía conseguiremos incentivar su motivación autodeterminada hacia el ejercicio y por lo tanto, disponer de una alta probabilidad de adherencia a la práctica deportiva futura.

\section{Referencias}

Adie, J. W.; Duda, J. L., \& Ntoumanis, N. (2012). Perceived coach-autonomy support, basic need satisfaction and the well- and ill-being of elite youth soccer players: A longitudinal investigation. Psychology of Sport and Exercise, 13, 51-59. http://dx.doi.org/10.1016/j.psychsport.2011.07.008

Álvarez, M. S.; Balaguer, I.; Castillo, I., \& Duda, J. L. (2009). Coach autonomy support and quality of sport engagement in young soccer players. The Spanish Journal of Psychology, 12, 138-148.

http://dx.doi.org/10.1017/S1138741600001554

Alves, S.; Franco, S.; Castañer, M.; Camerino, O.; Rodrigues, J., y Hileno, R. (2015). El análisis de la comunicación paraverbal cinésica y proxémica de los instructores de fitness mediante patrones temporales (T-patterns). Cuadernos de Psicología del Deporte, 15(1), 111-122.

Anguera, M.T.; Camerino, O.; Castañeñr, M., \& Sánchez-Algarra, P. (2014). Mixed methods en la investigación de la actividad física y deporte. Revista de Psicología del Deporte, 23(1), 123-130.

Bartholomew, K. J.; Ntoumanis, N.; Ryan, R. M.: Bosch, J. A., \& Thogersen-Ntoumani, C. (2011). Self-determination theory and diminished functioning: The role of interpersonal control and psychological need thwarting. Personality and Social Psychology Bulletin, 37(11), 1459-1473.

http://dx.doi.org/10.1177/0146167211413125

Chatzisarantis, N. L.; Hagger, M. S.; Smith, B., \& Wang, C. K. (2003). A meta-analysis of perceived locus on causality in exercise, sport, and physical education contexts. Journal of Sport and Exercise Psychology, 25(3), 284-306.

Condon, R., \& Collier, C. S. (2002). Student choice makes a difference in physical education. Journal of Physical Education, Recreation \& Dance, 73(2), 26-30. http://dx.doi.org/10.1080/07303084.2002.10607750

Conroy, D. E., \& Coatsworth, J. D. (2007). Assessing autonomy-supportive coaching strategies in youth sport. Psychology Sport Exercise Journal, 8(5), 671-684. http://dx.doi.org/10.1016/j.psychsport.2006.12.001

Creswell, J.W., \& Plano Clark, V.L. (2007). Designing and conducting mixed methods research. Thousand Oaks, CA: Sage.

Deci, E. L., \& Ryan, R. M. (1985). Intrinsic motivation and self-determination in human behavior. NY, USA: Plenum Press.

http://dx.doi.org/10.1007/978-1-4899-2271-7

Deci, E. L., \& Ryan, R. M. (1991). A motivational approach to self: Integration in personality. In R. Dienstbier, (Ed.), Nebraska Symposium on Motivation: Perspectives on motivation (Vol. 38, pp. 237-288). Lincoln, NE: University of Nebraska Press. 
Puigarnau, S.; Camerino, O.; Castañer, M.; Prat, Q.; Anguera, M.T. (2016). El apoyo a la autonomía en practicantes de centros deportivos y de fitness para aumentar su motivación. RICYDE. Revista internacional de ciencias del deporte, 43(12), 48-64. http://dx.doi.org/10.5232/ricyde2016.04303

Deci, E. L., \& Ryan, R. M. (2000). The "what" and "why" of goal pursuits: Human needs and the self-determination of behaviour. Psychological Inquiry, 11(4), 227-268. http://dx.doi.org/10.1207/S15327965PLI1104_01

Edmunds, J.; Ntoumanis, N., \& Duda, J. L. (2008). Testing a self-determination theory based teaching style in the exercise domain. European Journal of Social Psychology, 38(2), 375-388.

http://dx.doi.org/10.1002/ejsp.463

Gerring, J. (2007). Case study research. Principles and practices. Cambridge: Cambridge University Press.

Hagger, M. S.; Chatzisarantis, N. L., \& Culverhouse, T. (2003). The process by which perceived autonomy support in physical education promotes leisure-time physical activity intentions and behavior. Journal of Educational Psychology, 95(4), 784-795. http://dx.doi.org/10.1037/0022-0663.95.4.784

Jolly, K.; Duda, J. L.; Daley, A., Eves, F.; Nanette, M., Ntoumanis, N.; Rouse, P.; Lodhia, R., \& Williams, G. C. (2009). Evaluations of a standard provision versus an autonomy promotive exercise referral programme: rationale and study design. BMC Public Health, 9, 176-184.

http://dx.doi.org/10.1186/1471-2458-9-176

King A.C. (2001). Interventions to promote physical activity in older adults. The Journals of Gerontology, 56, 36-46.

http://dx.doi.org/10.1093/gerona/56.suppl_2.36

Kinnafick, F. E.; Thogersen-Ntoumani, C.; Duda, J. L., \& Taylor, I. (2014). Sources of autonomy support, subjective vitality and physical activity behavior associated with participation in a lunchtime walking intervention for physically inactive adults. Psychology of Sport and Exercise, 15(2), 190-197. http://dx.doi.org/10.1016/j.psychsport.2013.10.009

Leyton, M.; Jiménez, R.; Naranjo, J.; Castillo, M., y Morenas, J. (2013). Aplicación de un programa motivaciona para promocionar la actividad física en adultos y mayores. Archivos de Medicina del Deporte, 30(1), 25-33.

Moreno, J. A., y Martínez, A. (2006). Importancia de la teoría de la Autodeterminación en la práctica físico-deportiva: fundamentos e implicaciones prácticas. Cuadernos de Psicología del deporte, 6(2), 39-54.

Moreno, J. A.; Águila, C., y Borges, F. (2011). La socialización en la práctica físicodeportiva de carácter recreativo: predictores de los motivos sociales. Apunts. Educació Física i Esports, 103, 76-82.

Moustaka, F. C., Vlachopoulos, S. P., Kabitsis, C., \& Theodorakis, Y. (2012). Effects of an autonomy-supportive exercise instructing style on exercise motivation, psychological well-being, and exercise attendance in middle-age women. Journal of Physical Activity \& Health, 9(1), 138-150.

Ng, J. Y. Y.; Ntoumanis, N.; Thogersen-Ntoumani, C.; Stott, K., \& Hindle, L. (2013). Predicting psychological needs and well-being of individuals engaging in weight management. The role of important others. Applied Psychology: Health and WellBeing, 5, 291-310.

http://dx.doi.org/10.1111/aphw.12011

Ommundsen, Y., \& Kvalo-Eikanger, S. (2007). Autonomy-mastery, supportive or performance focused? Different teacher behaviours and pupils' outcomes in physical education. Scandinavian Journal of Medicine and Science in Sport, 51(4), 358-413. http://dx.doi.org/10.1080/00313830701485551 
Puigarnau, S.; Camerino, O.; Castañer, M.; Prat, Q.; Anguera, M.T. (2016). El apoyo a la autonomía en practicantes de centros deportivos y de fitness para aumentar su motivación. RICYDE. Revista internacional de ciencias del deporte, 43(12), 48-64. http://dx.doi.org/10.5232/ricyde2016.04303

Pope, J. P., \& Wilson, P. M. (2012). Understanding motivational processes in university rugby players: A preliminary test of the hierarchical model of intrinsic and extrinsic motivation at the contextual level. International Journal of Sports Science and Coaching, 7, 89-107.

http://dx.doi.org/10.1260/1747-9541.7.1.89

Prochaska, J. O., \& DiClemente, C. C. (1983). Stages ans processes of self-change of smoking. Towards an integrative model of change. Journal of Consulting and Clinical Psychology, 51(3), 390-395.

http://dx.doi.org/10.1037/0022-006X.51.3.390

Prochaska, J. O.; DiClemente, C. C., \& Norcross, J. C. (1992). In search of the structure of behavior change. In J.D. Fisher, J.M. Chensly \& A. Nadler (Eds.), Initiating selfchanges: Social Psychological and Clinical Perspectives. New York: Springer-Verlag.

Prochaska, J.O., \& Marcus, B.H. (1994). The transtheoretical model: Applications to exercise. In R.K. Dishman (Ed.), Advances in exercise adherence (pp. 161-180). Champaign, IL: Human Kinetics.

Puente, R., \& Anshel, M. H. (2010). Exercisers' perceptions of their fitness instructor's interacting style, perceived competence, and autonomy as a function of selfdetermined regulation to exercise, enjoyment, affect, and exercise frequency. Scandinavian Journal of Psychology, 51(1), 38-45. http://dx.doi.org/10.1111/j.1467-9450.2009.00723.x

Rahman, R. J.; Thogersen-Ntoumani, C.; Thatcher, J., \& Doust, J. (2011). Changes in need satisfaction and motivation orientation as predictors of psychological and behavioural outcomes in exercise referral. Psychology \& Health, 26(11), 1521-1539. http://dx.doi.org/10.1080/08870446.2010.538849

Sallis, J. F., \& Owen, N. (1999). Physical activity and behavioral medicine. Thousand Oaks, CA: Sage.

Silva M. N.; Markland, D. A.; Minderico, C. S.; Vieira, P. N.; Castro, M. M.; Coutinho, S. R.; Santos, T. C.; Matos, M. G.; Sardinha, L. B., \& Teixeira, P. (2008). A randomized controlled trial to evaluate self-determination theory for exercise adherence and weight control: Rationale and intervention description. BMC Public Health, 8, 234247.

http://dx.doi.org/10.1186/1471-2458-8-234

Stake, R.E. (1998). Investigación con estudio de casos. Madrid: Morata.

Stake, R.E. (1994). Case Studies. In N.K. Denzin y Y.S. Lincoln (Eds), Hanbook of qualitative research (pp. 236-247). Thousand Oaks, CA: Stage Publications.

Standage, M., Duda, J. L., \& Ntoumanis, N. (2003). A model of contextual motivation in physical education: Using constructs and tenets from self-determination and goal perspective theories to predict physical activity intentions. Journal of Educational Psychology, 95(1), 97-110.

http://dx.doi.org/10.1037/0022-0663.95.1.97

Standage, M.; Duda, J. L., \& Ntoumanis, N. (2005). A test of self-determination theory in school physical education. British Journal of Educational Psychology, 75(3), 411-433.

Standage, M.; Gillison, F., \& Treasure, D.C. (2007). Self-determination and motivation in physical education. In M. S. Hagger, \& N. L. D. Chatzisarantis (Eds.), Selfdetermination theory in exercise and sport (pp. 71-85). Champaign, IL: Human Kinetics.

Surbeck, E.; Han, E. \& Moyer, J. (1991). Assessing Reflective Responses in Journals. Educational Leadership, 48(6), 25-27. 
Puigarnau, S.; Camerino, O.; Castañer, M.; Prat, Q.; Anguera, M.T. (2016). El apoyo a la autonomía en practicantes de centros deportivos y de fitness para aumentar su motivación. RICYDE. Revista internacional de ciencias del deporte, 43(12), 48-64. http://dx.doi.org/10.5232/ricyde2016.04303

Vallerand, R. J. (2001). A hierarchical model of intrinsic and extrinsic motivation in sport and exercise. In G.C. Roberts (Ed.), Advances in motivation in sport and exercise (pp. 263-319). Champaign, IL: Human Kinetics.

Vallerand, R. J. (2007a). Intrinsic and extrinsic motivation in sport and physical activity: A review and a look at the future. In G. Tenenbaum, \& R. E. Eklund (Eds.), Handbook of sport psychology ( $3^{\text {rd }}$ ed., pp. 49-83) New York: John Wiley. http://dx.doi.org/10.1002/9781118270011.ch3

Vallerand, R. J. (2007b). A hierarchical model of intrinsic and extrinsic motivation for sport and physical activity. In M. S. Hagger, \& N. L. D. Chatzisarantis (Eds.), Selfdetermination theory in exercise and sport (pp. 255-279). Champaign, IL: Human Kinetics.

Williams, G. C.; Lynch, M. F.; McGregor, H. A.; Ryan, R. M.; Sharp, D., \& Deci, E. L. (2006). Validation of the "important other" climate questionnaire: assessing autonomy support for health-related change. Families, Systems \& Health, 24(2), 179-194.

http://dx.doi.org/10.1037/1091-7527.24.2.179

Williams, G. C.; McGregor, H. A.; Sharp, D.; Levesque, C.; Kouides, R. W., \& Ryan, R. M. (2006). Testing a self-determination theory intervention for motivating tobacco cessation: supporting autonomy and competence in a clinical trial. Health Psychology, 25(1), 91-101.

http://dx.doi.org/10.1037/0278-6133.25.1.91

Wilson, P.H.; Kaplan, B.J.; Crawford, S.G., \& Roberts, G. (2007). The Developmental Coordination Disorder Questionnaire 2007. Calgary, Canada: Alberta Children's Hospital Decision Support Research Team.

Yin, R.K. (2014). Case study research, design and methods, ( $5^{\text {th }}$ ed.). Beverly Hills, CA: Sage.

Zabalza, M. A. (2008). Diarios de clase: un instrumento de investigación y desarrollo profesional. Madrid: Narcea. 
Puigarnau, S.; Camerino, O.; Castañer, M.; Prat, Q.; Anguera, M.T. (2016). El apoyo a la autonomía en practicantes de centros deportivos y de fitness para aumentar su motivación. RICYDE. Revista internacional de ciencias del deporte, 43(12), 48-64. http://dx.doi.org/10.5232/ricyde2016.04303

\section{Anexo 1: consentimiento informado de participación en el estudio}

Su participación en este proyecto de investigación es totalmente voluntaria. Está en su derecho de abandonarlo en cualquier momento avisando debidamente a los responsables. La duración total del estudio es de nueve meses ( 6 meses en la práctica), durante los que seguirá un programa de actividad física, de 3 sesiones semanales, a la vez que realizará los registros y cuestionarios pertinentes.

Formar parte del estudio le otorga unos derechos pero también supone unas obligaciones.

Como participante del actual estudio, dispondrá del acceso gratuito a las instalaciones Olimpia Sport de Tárrega y de un seguimiento profesional durante las horas pactadas. Este vínculo finalizará una vez se haya completado el tiempo del estudio.

Las obligaciones como participante serán: asistir a las sesiones programadas con puntualidad, responder los cuestionarios y rellenar el diario de evolución, respetar el funcionamiento de la instalación colaborada e informar de cualquier imprevisto que pueda suponer un impedimento para seguir participando en el programa. Si el comportamiento de cualquier participante se considera que entorpece la evolución del estudio, los investigadores tendrán el derecho de dar por finalizada su participación.

La información que facilitará durante la duración del estudio serán confidenciales en las entidades involucradas. Las fotografías y registros realizados se utilizarán como datos del estudio, sin que ser utilizados por otros fines ajenos al estudio. Los resultados de esta investigación pueden ser publicados en revistas científicas o ser presentadas en reuniones médicas preservando la identidad del participante.

Debido a que cualquier problema de salud puede suponer una complicación, le pedimos que realice una revisión médica previa al inicio del programa donde se indique la capacidad de realizar actividad física de intensidad moderada.

He leído la información de este documento de consentimiento y autorizo el uso y divulgación de mi información de salud a las entidades mencionadas con anterioridad para los propósitos descritos en el consentimiento.

Al firmar esta hoja de consentimiento, no se renuncia a ningún de los derechos legales.

\section{Gracias}

[Firma del participante]

Nombre del

participante: Fecha: 